\title{
BMJ Open Why do some people with type 2 diabetes who are using insulin have poor glycaemic control? A qualitative study
}

\author{
Wen Ting Tong, ${ }^{1}$ Shireene Ratna Vethakkan, ${ }^{2}$ Chirk Jenn $\mathrm{Ng}^{1}$
}

To cite: Tong WT, Vethakkan SR, Ng CJ. Why do some people with type 2 diabetes who are using insulin have poor glycaemic control? A qualitative study. BMJ Open 2015;5:e006407. doi:10.1136/ bmjopen-2014-006407

- Prepublication history for this paper is available online. To view these files please visit the journal online (http://dx.doi.org/10.1136/

Received 19 August 2014 Revised 14 October 2014 Accepted 15 October 2014 bmjopen-2014-006407).

\section{ABSTRACT}

Objective: To explore factors influencing poor glycaemic control in people with type 2 diabetes using insulin.

Research design: A qualitative method comprising indepth individual interviews. A semistructured interview guide was used. The interviews were audiorecorded, transcribed verbatim and analysed using a thematic approach.

Participants: Seventeen people with type 2 diabetes using insulin with glycated haemoglobin $(\mathrm{HbA} 1 \mathrm{C}) \geq 9 \%$ for $>1$ year.

Setting: The Primary Care Clinic and Diabetes Clinic in the University of Malaya Medical Centre (UMMC), Malaysia.

Results: Data analysis uncovered four themes: lifestyle challenges in adhering to medical recommendations; psychosocial and emotional hurdles; treatment-related factors; lack of knowledge about and self-efficacy in diabetes self-care.

Conclusions: Factors that explain the poor glycaemic control in people with type 2 diabetes using insulin were identified. Healthcare providers could use these findings to address patients' concerns during consultations and help to improve glycaemic control.

\section{INTRODUCTION}

Insulin has been identified as the most effective glucose-lowering agent; however, it has been shown that many people with diabetes who are using insulin still fail to achieve glycaemic control. ${ }^{12}$

The challenges of achieving glycaemic

${ }^{1}$ Faculty of Medicine Department of Primary Care Medicine, University of Malaya, Kuala Lumpur, Malaysia

${ }^{2}$ Faculty of Medicine, Department of Medicine, University of Malaya, Kuala Lumpur, Malaysia

Correspondence to Wen Ting Tong; wentingtong@yahoo.com control in people with diabetes using insulin are: the progression of the disease; the impact of hypoglycaemia and weight gain; the burden of polypharmacy; lack of resources in provision of diabetes self-care education and support of patients; and the inherent limitations of subcutaneous exogenous insulin administration. ${ }^{3}$ Other predictors of poor glycaemic control in people with type 2 diabetes using insulin include younger age, shorter duration of diabetes diagnosis, lower body mass index, and

\section{Strengths and limitations of this study}

- The major strength lies in the fact that reasons for poor glycaemic control were uncovered from the perspectives of people with type 2 diabetes with sustained hyperglycaemia for more than 1 year despite insulin use, through in-depth interviews.

- Issues such as difficulty of adhering to regular meal and medication times, fear of hypoglycaemia, needles and pain, and lack of knowledge about and self-efficacy in diabetes care were found to be barriers to glycaemic control in people with type 2 diabetes using insulin.

- Issues such as social stigma, ethnicity, socioeconomic factors, family, friends, healthcare system and healthcare providers did not emerge as reasons for poor glycaemic control despite insulin use.

- The recruitment of participants was conducted in a single hospital, and therefore the involvement of healthcare systems in poor glycaemic control cannot be further explored.

- The fact that the interviews were conducted in the hospital environment may have influenced the participants to give a socially desirable response. However, they were informed that their responses would not affect their medical care and would be kept confidential.

poor physical functioning. ${ }^{4}$ Barriers to glycaemic control highlighted in a qualitative study of people with type 2 diabetes using insulin were fear about illness, guilt or selfblame, shame, ideas or beliefs about causation of diabetes, personal or cultural beliefs, and difficulty finding common ground with clinicians on diabetes management. ${ }^{5}$

To date, many studies have been conducted on barriers to insulin initiation. ${ }^{6-8}$ Research on factors associated with poor glycaemic control in people with type 2 diabetes has been largely quantitative and tended to focus on specific treatment modalities such as lifestyle modifications, oral hypoglycaemic agents (OHAs), OHAs+insulin, and insulin only. ${ }^{9-11}$ Qualitative studies have mainly focused on barriers to diabetes self-care 
management in general $^{12-15}$ rather than reasons for poor glycaemic control. Very few qualitative studies have examined factors affecting poor glycaemic control from the patient's perspective, especially among those with type 2 diabetes with poor glycaemic control despite using insulin.

Since insulin is the most effective glucose-lowering agent, it is pertinent to understand from the patient's perspective why patients with type 2 diabetes who are on insulin still fail to achieve glycaemic control. This study will help fill the gap in the existing literature by exploring factors influencing poor glycaemic control in people with type 2 diabetes using insulin. An understanding of the barriers to achieving glycaemic control will help healthcare providers (HCPs) find ways to improve glycaemic control in this subpopulation.

\section{RESEARCH DESIGN AND METHODS}

This study used a qualitative methodology comprising in-depth individual interviews to help understand patients' experiences, as well as taking into account the circumstances that led to poor glycaemic control in people with type 2 diabetes using insulin.

This study was conducted at the Primary Care Clinic and the Diabetes Clinic in the University of Malaya Medical Centre (UMMC). We purposively sampled patients who were diagnosed with type 2 diabetes, using insulin, either alone or in combination with OHAs, and with poor control of diabetes (glycated haemoglobin (HbA1c) $\geq 9 \%$ ) for at least 1 year. Participants were chosen from various sociodemographic backgrounds (age, ethnicity, education level) so that different perspectives on the reasons for poor glycaemic control could be explored.

We used a semistructured interview guide (box 1), which was developed based on the study's conceptual framework (figure 1) drawn from literature review and expert opinion. We reviewed the literature to identify possible factors, concepts and variables ${ }^{16}$ that have been shown to influence glycaemic control in people with diabetes. A preliminary conceptual framework was developed based on these factors. Later, the conceptual framework was given to two researchers (CJN and SRV, one a family medicine specialist and the other an endocrinologist) to provide feedback and to strengthen the conceptual framework based on their clinical experience and expertise. Subsequently, the interview guide was constructed based on the revised conceptual framework.

The interviews were carried out between January and August 2013 in consultation rooms in both clinics. Written informed consent and sociodemographic information were obtained from patients who agreed to participate. During the interviews, participants were asked why they thought their blood sugar was not well controlled despite using insulin. When the participant could think of no more reasons, the researcher would then probe other areas contributing to poor glycaemic
Box 1 Summary of interview guide topics on factors influencing poor glycaemic control despite using insulin

PREAMBLE: You have had diabetes for a long time and are now using insulin to control your blood sugar. As you are using insulin to control your blood sugar, but your blood sugar is still not well controlled

- Can you tell me what you think the reasons are for your blood sugar still not being well controlled?

Focusing on areas influencing poor glycaemic control

- Do you have problems adjusting your lifestyle to your diabetes care? (Probe: diet, exercise, medications). How?

-What hurdles do you face when using insulin? (Probe: injecting insulin in public; negative beliefs about insulin; fear of needles, pain, blood, body injury, marks, scars, weight gain and hypoglycaemia; knowledge about and skills in administrating insulin).

- Do your family, friends or employer help with your diabetes management? Do you think they affect your blood sugar control? How?

- Do you have any health problems that make it difficult for you to manage your diabetes? (Probe: vision problems, dexterity, mobility, polypharmacy, exercise).

- There are some people with diabetes who are depressed and stressed, and this affects their sugar control. Do you face this problem? How does it affect you in controlling your blood sugar?

Do you perform self-monitoring of blood glucose? If no, why not? Does it affect your blood sugar control? If yes, how?

- What hurdles do you face when consulting the doctor/nurse about your diabetes? (Probe: language, communication and interaction). Does it affect your blood sugar control?

- What do you think of the hospitals and clinics that you go to for diabetes management? (Probe: resources, complexity of system, accessibility, long waiting time, short consultation time). Does it affect your blood sugar control?

- Do you face any financial difficulties in managing your diabetes? (Probe: medication cost, transportation to hospitals, self-monitoring of blood glucose).

control, as developed in the interview guide. Data saturation was achieved at the 17th interview, when no new factors influencing poor glycaemic control emerged from the interviews.

It is important to note that the participants were recruited from the clinics where SRV and CJN conduct their clinical practice. Thus, in order to offset the influence of power disparities between doctor and patient, all the interviews were conducted by WTT. WTT is competent in English, Malay and Cantonese, hence the interviews were conducted in three languages. Of the 17 interviews, two were conducted in Cantonese and seven in Malay. Given that the Cantonese language has many colloquialisms, the recordings were translated directly into English by WTT so the meaning would not be lost. Other interviews that were conducted in English and Malay were given to experienced transcribers for verbatim transcription. All the transcripts were checked for accuracy and quality by WTT by listening to the audio recording and checking against the transcript, before 


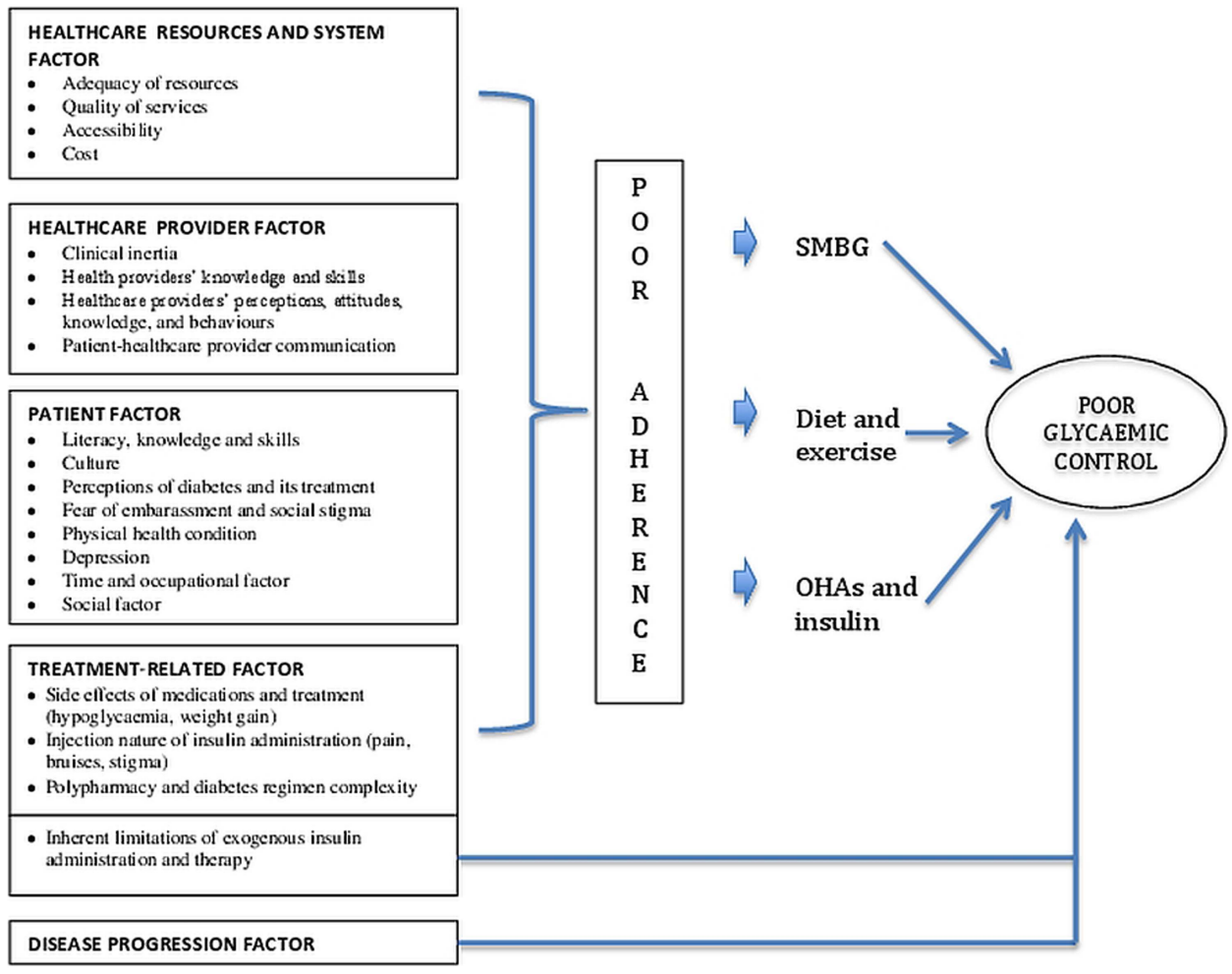

Figure 1 Conceptual framework of study. OHA, oral hypoglycaemic agent; SMBG, self-monitoring of blood glucose.

being exported into NVivo qualitative software for data analysis using a thematic approach. Malay transcripts were analysed in the said language, and the selected quotes were later translated into English. The translated quotes were checked by other researchers to ensure that the meaning was not lost or distorted.

Initially, the transcripts were read through for familiarisation by the researchers, and then codes were assigned to a particular phrase, sentence or paragraph that described the meaning of the text segment. Sentences that had a similar meaning were given the same code, while texts with a different meaning were given a new code. The whole transcript was analysed until there were no new meanings from the texts to form new codes. Subsequently, all the codes were compared, and related codes were clustered together under the same category. Irrelevant codes were omitted. The categories were later compared and further clustered under themes. The mapping of categories and themes resulted in the development of a coding frame. The coding frame was developed from the coding process on the first three transcripts by all the researchers (WTT, CJN, SRV). The coding frame was finalised when consensus was reached on the categories and themes. The finalised coding frame was used to code for the remaining transcripts by WTT. New emerging codes were added to the list of categories and themes created through constant discussion with other researchers to ensure the list of categories and themes produced the best representation of data obtained. Researchers constantly challenged one another's interpretation of the data to offset any potential biases when analysing the data.

\section{RESULTS}

Sociodemographic and diabetes profile of participants There were 17 participants in this study. Their sociodemographic and diabetes profiles are listed in table 1.

\section{Emerging themes}

Four themes that correspond to factors influencing poor glycaemic control despite insulin use emerged from the data analysis (table 2).

Table 1 Sociodemographic background and diabetes profile of the 17 participants

\begin{tabular}{ll}
\hline Characteristic & Value \\
\hline Age range (years) & $22-69$ \\
Sex, $\mathrm{n}$ & \\
$\quad$ Female & 10 \\
Male & 7 \\
Race, $\mathrm{n}$ & \\
$\quad$ Malays & 8 \\
Chinese & 4 \\
Indians & 4 \\
Nepalese & 1 \\
Education, $\mathrm{n}$ & \\
Secondary & 9 \\
Tertiary & 5 \\
Primary & 2 \\
$\quad$ No formal education & 1 \\
Years living with diabetes (range) & $2-30$ \\
Years using insulin (range) & $1-14$ \\
\hline
\end{tabular}


Table 2 Factors influencing poor glycaemic control in people with type 2 diabetes using insulin

\section{Theme}

1 Lifestyle challenges in adhering to medical recommendations

2 Psychosocial issues and emotional hurdles

3 Diabetes-treatment-related factors

4 Lack of knowledge about and self-efficacy in diabetes self-care

$\mathrm{HCP}$, healthcare provider.

\section{Category}

1. Difficulty integrating diabetes medical recommendations into work-life schedule

2. Inability to control food cravings and eating habits

3. Inappropriate diet recommendations by HCPs

4. Health-limiting conditions affecting exercise

1. Psychosocial problems affecting diabetes self-care management

2. Loss of motivation

3. Perceived poor glycaemic control as part of ageing

1. Side effects of insulin

2. Perception of appropriate dietary practices related to insulin

1. Lack of knowledge of glycaemic level and target

2. Lack of self-efficacy in adjustment of insulin dosage
Lifestyle challenges in adhering to medical recommendations

Four subthemes were identified.

\section{Difficulty integrating diabetes medical recommendations into work-life schedule}

Participants found it difficult to integrate medical recommendations, such as a medication regimen and meal times, when they did not match their schedule of daily activity. When participants were too busy with their work-life schedule, they tended to skip meals which caused them to become hungry and overeat later. Skipping meals also resulted in them missing or delaying their insulin injections.

The way I eat and take the medications is not consistent. Sometimes I forget. Maybe I am too busy. Every time my insulin use would be delayed. For example, usually we inject at 12 right, sometimes I will inject at 2. Sometimes I did not inject at all-58-year-old housewife

One participant described how the nature of his occupation made it difficult for him to adhere to a healthy diet and insulin treatment.

We are going around okay. So we can't just go and get what we want to eat. We can't go and pack something or bring the food from house. Furthermore, like now I'm taking the short-acting insulin, so every meal time you have to inject. You just can't go and take insulin, you see. I'm working as a bodyguard you see, you have to follow the boss closely. I think so that is the reason [for poor blood sugar].-36-year-old personal bodyguard

\section{Inability to control food cravings and eating habits}

Participants also reported that the temptation to eat something delicious would lead them to lose control of their diet, causing them to overeat.
My eating habit. Like I like to eat sweets, like kuihs [local dessert] and all that. But I have to control. I know I am not controlling. I must put a full stop to that.60-year-old housewife

It is also difficult to resist food when there is a variety of food available and coming from a lifestyle and culture where food and eating are a way of life.

Basically it is also Malaysia lifestyle whereby people like to eat. You eat non-stop. Sugar is particularly everywhere in your diet so that's probably one of the main reasons why it is not controlled.-22-year-old student

\section{Inappropriate diet recommendations by HCPS}

Participants felt that the diet recommended by HCPs provided insufficient energy for them to carry out their work. Some also expressed frustration about the monotony of eating the same types of food every day, such as bread and chapatti, which were recommended by the HCPs. Hence, they often neglected the dietary advice.

Every time they [HCPs] ask me to eat bread. Can you eat bread everyday? For sure you will hate it. They will ask you to eat vegetables every day. Cannot like that59-year-old ex-lorry driver

\section{Health-limiting conditions affecting exercise}

Not being able to exercise optimally due to health conditions was another reason cited by many for poor glycaemic control.

Another thing is exercise. Because of a stroke, I have problems with walking. I have to exercise more.-61-year-old engineer

Psychosocial issues and emotional hurdles Three subthemes emerged. 
Psychosocial problems affecting diabetes self-care management

Participants felt that their poor glycaemic control was attributable to personal problems which caused them to feel anxious, stressed and sad, which resulted in some adopting unhealthy eating habits and not taking their diabetes medications, including insulin.

Actually when you have diabetes, you cannot be stressed. Previously when I was under stress [due to marital problems], my blood sugar level was very high because I did not eat and take my insulin. I was hoping to die.50-year-old taxi driver

\section{Loss of motivation}

Participants admitted that they were so tired of adhering to diabetes medications after having taken them for such a long time that sometimes they would intentionally skip doses.

Sometimes I purposely miss them because I am just so tired of injecting.-40-year-old officer

In addition, an absence of significant improvements in glycaemic control despite efforts made led participants to 'give up' on controlling their blood sugar.

There's one time actually I did go to the gym and the exercise was okay but it didn't really do anything to my weight. It does a little bit on my sugar but after a while I just give up. I think it would be as well [contributed to her poor glycaemic control] because the main thing is that, I think that if I actually lose weight, I would be able to control my sugar as well.-40-year-old officer

\section{Perceived poor glycaemic control as part of ageing}

Many older participants in this study held the view that, whatever their attempts to control blood glucose levels, their glycaemic control would still fall short because of their advanced age.

Maybe because I am getting old. As the days passed by, all my organs have deteriorated. Like an engine, the more it is used, it will become spoilt.-69-year-old retiree

\section{Treatment-related factors}

Two subthemes were found.

\section{Side effects of insulin}

Participants reported that they would tend to overeat to prevent or counter the effects of insulin-induced hypoglycaemia. However, it is when participants overeat that their glycaemic control deteriorates.

I had a fit once (due to hypoglycaemia), that fear is always there. On and off, I used to eat more to make sure I don't go into hypoglycaemia fit. It is extremely painful. -47-year-old doctor
Participants also felt that insulin caused them to feel hungry, causing them to overeat, hence, raising their blood sugar levels.

But if I use insulin, it makes me eat. I feel that after using insulin, the blood sugar goes even higher.37-year-old clerk

Fear of needles and pain also caused participants to delay insulin initiation as well as intentionally skip injections, thus contributing to poor glycaemic control.

I don't quite like insulin actually. I'm very afraid of needles and the pain that follows. In a week I would say at least 3 times [skipping insulin injections]. Although my blood sugar was already up about 6 to 7 years ago, but I've only started insulin not far back from now. So that's the other reason [for poor glycaemic control].40-year-old officer

\section{Perception of appropriate dietary practices related to insulin} One participant felt that his poor glycaemic control could be attributed to the diet recommendations given by the HCP. He stated that the meal pattern recommended was not right and would instead reduce the efficacy of the insulin.

For example if you eat at $8 \mathrm{pm}$, then you feel hungry and you eat again. So if I follow his [doctor] advice I will eat but this is wrong. The mistake is if lets say I eat at $7 \mathrm{pm}$, then $8,9,10,11,12 \mathrm{pm}$, for about 4 hours I will keep on eating. So the insulin cannot fight with my diabetes. Because I have experienced this so I know. The recommended cannot work. My diabetes reach 20, 30 something.-50-year-old taxi driver

\section{Lack of knowledge about and self-efficacy in diabetes self-care}

Two subthemes were identified.

\section{Lack of knowledge of glycaemic level and target}

Lack of knowledge of their glycaemic level and target was also cited as a reason for poor glycaemic control, as participants were not aware to what extent they should control their blood sugar. This lack of knowledge was attributed to difficulties in performing self-monitoring of blood glucose (SMBG) for financial reasons, and some claimed that their HCPs did not inform them about their glycaemic levels and target.

I check less because sometimes when the needles are finished, I have to wait for my salary to buy. I check once a week but if I need to see the doctor then only I will check 4 times a day. Actually it is not enough. When I don't check, I cannot control my diet so that's why my blood sugar is not good.-37-year-old clerk

I don't know why he [doctor] wants to lower (blood sugar level) some more. No, because I don't know what 
is the target. The doctor never mentioned. I am also not sure. So I also don't know whether I am okay or not. If I know, I will control no matter what.-31-year-old marketing coordinator

\section{Lack of self-efficacy in adjustment of insulin dosage}

Despite receiving advice from the doctor that they could adjust their insulin dosage, some participants did not do so because they were afraid of making mistakes when adjusting the insulin dose, which could lead to hypoglycaemia and other complications.

I'm just reluctant [to adjust insulin dosage] because they [doctor] won't be with me 24 hours. I didn't increase or decrease any of the medication. I just stick to it. So maybe that is the reason.-36-year-old personal bodyguard

\section{DISCUSSION}

Our study revealed that people with type 2 diabetes using insulin attributed their sustained hyperglycaemia to lifestyle challenges, psychosocial and emotional problems, treatment-related factors, and lack of knowledge about glycaemic levels and targets, as well as poor selfefficacy with regard to insulin dosage adjustment. Most of the factors raised were not related to problems with insulin use per se, but were related to barriers to performing diabetes self-care tasks in general such as dietary control and adherence to exercise and medications including OHAs.

Our study participants faced difficulties in adhering to the recommended meal and insulin injection schedule because of work priorities and time constraints. In a multinational study involving 1530 people from eight countries with type 1 diabetes $(12.8 \%)$ and type 2 diabetes $(88.2 \%)$ using insulin, taking insulin at the prescribed time or with meals everyday was also reported to be difficult. ${ }^{17}$ The lifestyle changes required for diabetes management in terms of diet and regular meal times have been acknowledged to be hard to implement, even in people with type 2 diabetes using OHAs alone, who often report missed or delayed meals. ${ }^{18}$ This shows that adherence to regular meal and medication times is a universal and major barrier to diabetes management for people with diabetes. It is crucial to overcome this, especially for people with type 2 diabetes using insulin, as insulin administration has to be synchronised with meals. When regular meal times cannot be followed, it often results in delayed or skipped insulin intake, as reported in our study, which explains poor glycaemic control.

Our participants raised the issue of diets recommended by HCPs that did not meet their dietary needs; the issues were (a) the monotony of eating the same type of food every day and (b) the recommended diet did not provide sufficient energy. Other patients with type 2 diabetes using insulin have reported that clinicians would simply assume that they would comply with the medical recommendations, which were given to them without consideration of their individual needs and preferences. ${ }^{5}$
In addition, it also appears that lack of understanding of the rationale behind dietary recommendations is common among patients with type 2 diabetes. One participant in our study thought the meal pattern recommended by HCPs would thwart the efficacy of insulin, while, in another study, patients with type 2 diabetes using OHAs perceived that frequent meals was a way to control their diabetes. ${ }^{19}$ In fact, the main purpose of regular meals is to counter the effects of hypoglycaemia due to insulin and long-acting sulfonylureas. HCPs may be a contributing factor to these barriers to adhering to dietary recommendations. In a collaborative study conducted in Austria, Canada, Germany and the UK, it was found that general practitioners lack the knowledge and skills to educate, support and motivate patients on healthy lifestyle changes. ${ }^{20}$

The issue of psychosocial factors and lack of motivation is crucial, as it affects all aspects of diabetes self-care including adherence to insulin, as evidenced from our study. Diabetes self-care is a complex task that demands behavioural change in the patient on a daily basis; social, cultural, familial and professional influences further complicate management of the disease as shown in other studies. ${ }^{21-23}$ Furthermore, our participants also showed that, when a patient's diabetes condition remains unimproved despite efforts to control it, this leads to 'diabetes burn-out' stemming from frustration and loss of motivation, eventually resulting in neglect of diabetes self-care. Perhaps explaining to them the disease progression in type 2 diabetes and that progressive loss of $\beta$-cell function is common will lift the feeling of frustration and loss of motivation.

Older participants of our study perceived that they would never be able to achieve glycaemic control because of their age even with insulin use. Such misconceptions are alarming as they may decrease older people's perceived importance of glycaemic control. They may lower their expected treatment target in order to cope with the challenges of managing diabetes at such an age. ${ }^{24}$ There is a need to inform older people with type 2 diabetes that insulin has no upper dose limit and they will still be able to control their glycaemic levels even with increasing age.

Issues such as fear of hypoglycaemia, needles and pain are well-established barriers to insulin initiation, ${ }^{67}$ and it is interesting that such problems still prevail even after participants initiate insulin use, as found in our study. Moreover, these are well-established factors in intentional insulin omission $^{25}$ and overeating to prevent insulin-induced hypoglycaemia. ${ }^{5}{ }^{26}$ The UMMC has an established specialised diabetes clinic with trained diabetes nurses to provide education and skills training in diabetes self-care to patients. Therefore, our study participants would have been educated and trained in techniques of insulin administration and ways to prevent and manage hypoglycaemia. In addition to providing diabetes education and skills training to people with type 2 diabetes who are using insulin, provision of counselling to address these fears is warranted. 
Lack of knowledge of glycaemic levels and targets was also a reason for poor glycaemic control in our participants. They were unsure to what extent they should control their glucose levels. The issue of lack of knowledge of glycaemic levels and targets in our study stemmed from lack of SMBG and perceived minimal feedback from HCPs. Our participants reported financial constraints in carrying out effective SMBG, as costs of SMBG supplies are not subsidised by the Malaysian government. The impact of economic factors on SMBG adherence has been reported in other studies to be an issue that limits glycaemic control. ${ }^{27}{ }^{28}$ In a study by Onwudiwe et $a l^{29}$ participants reported that they were not informed by their doctor of their target blood glucose levels and perceived that as a barrier to diabetes self-management. HCPs have a crucial role to play in discussing glycaemia results with their patients and formulating mutually agreed glycaemic targets. Kumpatla et $a \vec{l}^{0}$ showed that a knowledge of HbAlc and the target goal had a positive effect on maintaining better glycaemic control among people with type 2 diabetes.

Self-adjustment of insulin dose has been shown to be a technically complex procedure for people with type 1 diabetes, ${ }^{31}$ and people with diabetes spend most of their time managing their diabetes away from HCPs. It is thus not surprising that our participants were still apprehensive about self-adjustment of their insulin dose, for fear of developing hypoglycaemia. Dependent and deferential attitudes towards health professionals were cited as the reasons why patients with type 1 diabetes do not adjust their insulin dose, ${ }^{31}$ and this may also be the reason for failure to adjust insulin dosing among our participants. Furthermore, lack of skills to educate patients on how to monitor their glycaemic levels and adjust their insulin dose has also been found to be a common challenge faced by general practitioners. ${ }^{20}$

Some possible factors for poor glycaemic control as highlighted in the conceptual framework did not emerge in our study findings even when the participants were probed. The issue of social stigma was not raised by our participants as a reason for poor glycaemic control. We assumed that our participants had overcome this barrier to initiation of insulin, since they had been on insulin for at least 1 year; they also reported performing adaptive strategies such as finding a private place to inject when in public-for example, in the toilet or in their car. Ethnicity was also not raised as a factor in poor glycaemic control in this study. Instead, the participants described eating culture as a way of life for Malaysians in general. Therefore, participants of this study might have adapted to the 'Malaysian' culture whereby they share and practise the culture of others. Even if the diet recommended by HCPs may not be the type of food familiar to the specific ethnic group or culture, they could still follow the recommended diet. No specific ethnicity barrier was also reported for diabetes treatment aspects. Socioeconomic factors were not a barrier for participants in this study to seeking healthcare treatment, as the company where they or their spouses worked subsidised the medical costs. It should also be noted that the Malaysian government provides relatively cheap healthcare for the population and the cost of insulin is subsidised. However, this is not the case for SMBG, where patients have to pay for a glucometer and test strips. This is why a lack of knowledge of glycaemic status because of low utilisation of SMBG was raised as a reason for poor glycaemic control. Our participants did not blame their family, friends, healthcare system or HCPs for their poor glycaemic control. They, however, expressed dismay at the short consultation times and not being able to see the same doctor each time about their diabetes. Our participants stated that diabetes control is a personal responsibility, and they therefore tended to focus on their personal inadequacies when it came to poor glycaemic control. This may be due to diabetes self-care playing a huge role in disease control, and hence people with type 2 diabetes may feel greater responsibility for self-care. Thus, when glycaemic control cannot be achieved, this results in self-blame. ${ }^{32}$

\section{Strengths and limitations of the study}

The major strength of this study lies in the fact that the reasons behind poor glycaemic control were gained from insights of people with type 2 diabetes with sustained hyperglycaemia for more than 1 year despite insulin use, through in-depth interviews to explain why their diabetes remains poorly controlled despite taking insulin. To our knowledge, such findings have not previously been reported.

This study found that issues such as the difficulty of adhering to regular meal and medication times, fear of hypoglycaemia, needles and pain, and lack of knowledge about and self-efficacy in diabetes care remain factors for poor glycaemic control in people with type 2 diabetes using insulin, whereas issues such as social stigma, ethnicity, socioeconomic factors, family, friends, healthcare system factors and HCPs were found not to be reasons for poor glycaemic control despite insulin use.

This study has a few limitations. The recruitment of participants was only conducted in a single hospital, and hence healthcare systems as a factor in poor glycaemic control cannot be further explored. The interviews were conducted in the hospital where the participants were recruited, and hence the environment may have influenced them to give a socially desirable response. However, they were informed that their responses would not affect their medical care and would be kept confidential.

\section{Clinical recommendations}

HCPs should create individualised plans in consultation with the patients to ensure a routine that allows for proper meal times and exercise enabling them to take their diabetes medication, including insulin, in a timely manner. Patients reported that they face problems with treatment recommendations; hence HCPs should continuously assess the efficacy and feasibility of treatment 
provided to their patients and clarify patient misconceptions. It is also pertinent for HCPs to recognise the psychological and emotional problems that impact on their patients' diabetes self-care and provide them with effective support. Lastly, HCPs should discuss glycaemic readings and adjustment of insulin dosage, as well as formulate a mutually agreed target with patients to facilitate improvement in glycaemic control.

Murray et $a t^{20}$ have identified the common challenges faced by general practitioners when caring for people with type 2 diabetes across international and health system borders; they are related to knowledge, skills, attitudes, behaviours and context. Some of the challenges faced by HCPs may explain the reasons for poor glycaemic control as faced by participants of this study such as the lack of knowledge and skills to: give clear explanations to their patients; actively engage their patients in their health management; educate patients on how to monitor their glycaemic levels; engage in shared decision-making with patients; and provide support and motivation to patients in their efforts to make lifestyle changes to achieve better glycaemic control. Therefore, it is pertinent that HCPs are equipped with the latest accurate knowledge and skills related to diabetes and its treatment and be able to impart them to their patients to empower them to perform effective diabetes self-care tasks.

\section{Future research recommendations}

More research on this topic is needed to uncover other factors that may influence poor glycaemic control despite insulin use. In addition, exploring views of HCPs and patients' caregivers would provide a more holistic understanding of factors involved in poor glycaemic control despite insulin use. Accurate assessment of a patient's knowledge, actual self-care practices, and clinical characteristics could also be conducted. This would allow triangulation of multiple sources of data, which would provide more comprehensive understanding and better identification of reasons for poor glycaemic control. Ultimately, the factors identified may help to develop a tool to be used by HCPs, such as a checklist to address the barriers to achieving glycaemic control faced by people with type 2 diabetes using insulin. In addition, future studies should look into the motivators of better glycaemic control in insulin-using patients with type 2 diabetes who have been successful in gaining glycaemic control. Understanding both barriers and motivators would help to improve glycaemic control in this subpopulation.

\section{CONCLUSIONS}

Our findings reveal lifestyle challenges, psychosocial and emotional problems, treatment-related factors, lack of knowledge of glycaemic levels and targets, and poor selfefficacy with regard to insulin dosage adjustment as factors in poor glycaemic control despite insulin use. HCPs should look into these factors and help insulin-using patients with type 2 diabetes to address their concerns during consultations and thus improve glycaemic control.

Contributors All authors (WTT, SRV, CJN) of this study conceived and designed the study, researched and analysed the data, contributed to discussion, and wrote, edited, reviewed and approved the final version of the manuscript.

Funding We thank the University of Malaya, Malaysia for funding this project (reference: PV109-2012A).

Competing interests None.

Ethics approval This study received ethics approval from the University of Malaya Medical Centre Medical Ethics Committee (926.18)

Provenance and peer review Not commissioned; externally peer reviewed.

Data sharing statement No additional data are available.

Open Access This is an Open Access article distributed in accordance with the Creative Commons Attribution Non Commercial (CC BY-NC 4.0) license, which permits others to distribute, remix, adapt, build upon this work noncommercially, and license their derivative works on different terms, provided the original work is properly cited and the use is non-commercial. See: http:// creativecommons.org/licenses/by-nc/4.0/

\section{REFERENCES}

1. Cramer JA, Pugh MJ. The influence of insulin use on glycemic control. Diabetes Care 2005;28:78-83.

2. Harris SB, Kapor J, Lank CN, et al. Clinical inertia in patients with T2DM requiring insulin in family practice. Can Fam Physician 2010;56:e418-24.

3. Davies M. The reality of glycaemic control in insulin treated diabetes: defining the clinical challenges. Int J Obes Relat Metab Disord 2004;28:S14-22.

4. Nichols GA, Hillier TA, Javor K, et al. Predictors of glycemic control in insulin-using adults with type 2 diabetes. Diabetes Care 2000;23:273-7.

5. Janes R, Titchener J, Pere J, et al. Understanding barriers to glycaemic control from the patient's perspective. J Prim Health Care 2013;5:114-22.

6. Abu Hassan $\mathrm{H}$, Tohid $\mathrm{H}$, Mohd Amin R, et al. Factors influencing insulin acceptance among type 2 diabetes mellitus patients in a primary care clinic: a qualitative exploration. BMC Fam Pract 2013;14:164.

7. Chen KW, Tseng HM, Huang YY, et al. The barriers to initiating insulin therapy among people with Type 2 diabetes in Taiwan-a qualitative study. J Diabetes Metab Disord 2012;3:194.

8. Lee YK, Lee PY, Ng CJ. A qualitative study on healthcare professionals' perceived barriers to insulin initiation in a multi-ethnic population. BMC Fam Pract 2012;13:28.

9. Sanal TS, Nair NS, Adhikari P. Factors associated with poor control of type 2 diabetes mellitus: a systematic review and meta-analysis. J Diabetol 2011;3:1-10.

10. Khattab M, Khader YS, Al-Khawaldeh A, et al. Factors associated with poor glycemic control among patients with Type 2 diabetes. J Diabetes Complications 2010;24:84-9.

11. Sasi ST, Kodali M, Burra KC, et al. Self care activities, diabetic distress and other factors which affected the glycaemic control in a tertiary care teaching hospital in South India. J Clin Diagn Res 2013;7:857-60.

12. Chlebowy DO, Hood S, LaJoie AS. Facilitators and barriers to self-management of type 2 diabetes among urban African American adults: focus group findings. Diabetes Educ 2010;36:897-905.

13. Shakibazadeh E, Larijani B, Shojaeezadeh D, et al. Patients' perspectives on factors that influence diabetes self-care. Iran J Public Health 2011;40:146-58.

14. Singh $H$, Cinnirella M, Bradley C. Support systems for and barriers to diabetes management in South Asians and Whites in the UK: qualitative study of patients' perspectives. BMJ Open 2012;2:e001459.

15. Hu J, Amirehsani K, Wallace DC, et al. Perceptions of barriers in managing diabetes: perspectives of Hispanic immigrant patients and family members. Diabetes Educ 2013;39:494-503.

16. Matthew BM, Huberman AM. Qualitative data analysis: an expanded sourcebook. SAGE Publications, 1994. 
17. Peyrot M, Barnett AH, Meneghini LF, et al. Insulin adherence behaviours and barriers in the multinational Global Attitudes of Patients and Physicians in Insulin Therapy study. Diabet Med 2012;29:682-9.

18. Frandsen KB, Smedegaard KJ. Compliance with, and understanding of, mealtime advice in patients with Type 2 diabetes. Diabetes 2000;49:a176.

19. Frandsen KB, Kristensen JS. Diet and lifestyle in type 2 diabetes: the patient's perspective. Pract Diabetes Int 2002;19:77-80.

20. Murray S, Lazure P, Schroter S, et al. International challenges without borders: a descriptive study of family physicians' educational needs in the field of diabetes. BMC Fam Pract 2011;12:27.

21. Samuel-Hodge CD, Headen SW, Skelly AH, et al. Influences on day-to-day self-management of type 2 diabetes among African-American women: spirituality, the multi-caregiver role, and other social context factors. Diabetes Care 2000;23:928-33.

22. Shacter HE, Shea JA, Akhabue E, et al. A qualitative evaluation of racial disparities in glucose control. Ethn Dis 2009;19:121-7.

23. Peyrot M, Rubin RR, Lauritzen T, et al. Psychosocial problems and barriers to improved diabetes management: results of the Cross-National Diabetes Attitudes, Wishes and Needs (DAWN) Study. Diabet Med 2005;22:1379-85.

24. Paul C, Ayis S, Ebrahim S. Disability and psychosocial outcomes in old age. J Aging Health 2007;19:723-41.
25. Peyrot $\mathrm{M}$, Rubin RR, Kruger DF, et al. Correlates of insulin injection omission. Diabetes Care 2010;33:240-5.

26. Perlmuter LC. Glycemic control and hypoglycemia. Is the loser the winner? Diabetes Care 2008;31:2072-6.

27. Ong WM, Chua SS, Ng CJ. Barriers and facilitators to self-monitoring of blood glucose in people with type 2 diabetes using insulin: a qualitative study. Patient Prefer Adherence 2014;8:237-46.

28. Yuan L, Guo X, Xiong Z, et al. Self-monitoring of blood glucose in type 2 diabetic patients in China: current status and influential factors. Chin Med J (Engl) 2014;127:201-7.

29. Onwudiwe NC, Mullins CD, Winston RA, et al. Barriers to self-management of diabetes: a qualitative study among low-income minority diabetics. Ethn Dis 2011;21:27-32.

30. Kumpatla S, Medempudi S, Manoharan D, et al. Knowledge and outcome measure of $\mathrm{HbA} 1 \mathrm{c}$ testing in Asian Indian patients with Type 2 diabetes from a tertiary care center. Indian J Community Med 2010;35:290-3.

31. Lawton J, Rankin D, Cooke D, et al. Patients' experiences of adjusting insulin doses when implementing flexible intensive insulin therapy: a longitudinal, qualitative investigation. Diabetes Res Clin Pract 2012:98:236-42.

32. Beverly EA, Ritholz MD, Brooks KM, et al. A qualitative study of perceived responsibility and self-blame in type 2 diabetes: reflections of physicians and patients. J Gen Intern Med 2012;27:1180-7. 